\title{
Fuzzy Bi-level Decision-Making Techniques: A Survey
}

\author{
Guangquan Zhang ${ }^{\mathrm{a}}$, Jialin $\mathrm{Han}^{\mathrm{a}, \mathrm{b}}$, Jie Lu ${ }^{\mathrm{a}}$ \\ ${ }^{a}$ Faculty of Engineering and Information Technology, University of Technology Sydney, NSW 2007, Australia \\ ${ }^{\mathrm{b}}$ School of Mechanical Engineering, Beijing Institute of Technology, Beijing 100081, China \\ E-mail: Guangquan.Zhang@uts.edu.au,Jialin.Han@student.uts.edu.au,Jie.Lu@uts.edu.au
}

Received 4 October 2015

Accepted 12 March 2016

\begin{abstract}
Bi-level decision-making techniques aim to deal with decentralized management problems that feature interactive decision entities distributed throughout a bi-level hierarchy. A challenge in handling bi-level decision problems is that various uncertainties naturally appear in decision-making process. Significant efforts have been devoted that fuzzy set techniques can be used to effectively deal with uncertain issues in bi-level decision-making, known as fuzzy bi-level decision-making techniques, and researchers have successfully gained experience in this area. It is thus vital that an instructive review of current trends in this area should be conducted, not only of the theoretical research but also the practical developments. This paper systematically reviews up-to-date fuzzy bi-level decisionmaking techniques, including models, approaches, algorithms and systems. It also clusters related technique developments into four main categories: basic fuzzy bi-level decision-making, fuzzy bi-level decision-making with multiple optima, fuzzy random bi-level decision-making, and the applications of bi-level decision-making techniques in different domains. By providing state-of-the-art knowledge, this survey paper will directly support researchers and practitioners in their understanding of developments in theoretical research results and applications in relation to fuzzy bi-level decision-making techniques.
\end{abstract}

Keywords: Bi-level decision-making, bi-level programming, fuzzy sets, fuzzy systems, decision support systems.

\section{Introduction}

Bi-level decision-making techniques, mainly implemented by bi-level programming, motivated by Stackelberg game theory [1], have been developed to address compromises between two interactive decision entities that are distributed throughout a hierarchical organization. In a bi-level decision-making process, decision entities at the upper level and the lower level are respectively termed the leader and the follower, and make their individual decisions in sequence with the aim of optimizing their respective objectives. The original appearance of bi-level decision-making was proposed by Bracken and McGill [2]. A wide range of research in this area has been undertaken with the designations bi-level programming, bi-level optim- ization, and bi-level decision-making. Bi-level decisionmaking mainly is a bi-level optimization issue and mainly applies bi-level programming methods. It often appears in many decentralized management problems, and has motivated a number of researches to work on bi-level decision models [3, 4], solution approaches [5, 6] and applications [7, 8].

A challenge in handling bi-level decision problems is that various uncertainties (including fuzziness and randomness) have naturally appeared in its decisionmaking process. Since uncertainty issue could function in both model parameter determination and solution process, information related to decision-making becomes highly imprecise and ambiguous, especially in the current age of big data. To tackle bi-level decision problems under uncertainty, fuzzy sets and fuzzy systems have been used in terms of both decision 
modeling and solution approaches, formed fuzzy bi-level decision-making techniques. To help researchers understand the development experience of fuzzy bilevel decision-making techniques, and to assist practitioners to handle related decision-making problems in practice, this paper reviews the latest research on fuzzy bi-level decision-making including theoretical research results and applications. It clusters the literatures into four research directions: (1) basic fuzzy bi-level decision-making, (2) fuzzy bi-level decision-making with multiple optima, (3) fuzzy random bi-level decision-making, and (4) applications of fuzzy bi-level decision-making techniques.

Several survey papers on bi-level programming/ decision-making have been published in the past 20 years. However, almost the survey papers, Ben-Ayed [9], Vicente and Calamai [10], and Colson et al. [11, 12] only presented overviews of bi-level decision-making, which focused on basic solution concepts and approaches, without addressing uncertain issues and fuzzy bi-level techniques. Paper by Sakawa and Nishizaki [5] is the only paper which reviewed fuzzy bilevel decision-making techniques. However, this paper only focused on interactive fuzzy programming approaches in terms of solving a range of stochastic bilevel and multilevel decision problems, and didn't examine the real-world application developments of fuzzy bi-level decision-making. Thus, to produce our paper is a significant work to give an overview of recent developments in fuzzy bi-level decision-making techniques.

Two main types of articles reviewed in this survey: One consists of theoretical research in fuzzy bi-level decision-making; and the other consists of research on fuzzy bi-level decision-making applications including related decision support systems and case studies. The literature search and selection were performed according to the following four steps:

Step 1. Publication database identification and determination: Publication databases, Science Direct, ACM Digital Library, IEEE Xplore and SpringerLink, were used to provide a comprehensive bibliography of research papers to this study.

Step 2. Preliminary screening of articles: Based on related keywords of fuzzy bi-level decision-making, the articles were then selected as references if they satisfied one of the following criteria that they: (1) proposed decision models related to fuzzy bi-level decision- making; (2) developed solution concepts and approaches for solving fuzzy bi-level decision problems; (3) provided a decision support system tool or a real-world application in relation to fuzzy bi-level decision-making.

Step 3. Theoretical research article selection: These papers were selected according to three criteria: (1) novelty - published within the last decade; (2) impact published in high quality (high impact factor) journals, or in conference proceedings or book chapters but with high citations; and (3) typicality - only the most typical decision models and solution approaches were retained. These papers are mainly used in Sections $2-4$ of this paper.

Step 4. Application research article selection: Theses papers were selected according to three criteria: (1) novelty, (2) impact, and (3) coverage - reported a new or particular application domain. These papers are mainly used in Section 5 .

The main contributions of this paper are threefold. First, the paper comprehensively and perceptively summarizes research achievements on fuzzy bi-level decision-making techniques from the point of view of theoretical research results and applications. Second, for each fuzzy bi-level decision-making technique, it carefully analyzes typical decision models and identifies the specific requirements for solution approaches; this will directly motivate and support researchers and practitioners to promote the popularization and application of fuzzy bi-level decision-making techniques in different domains. Third, it particularly suggests several very innovative emerging research topics/directions in the area of fuzzy bi-level decisionmaking.

The remainder of this paper is structured as follows. In Section 2, the techniques for basic fuzzy bi-level decision-making are reviewed. Section 3 presents technique development for fuzzy bi-level decisionmaking with multiple optima. Section 4 addresses fuzzy random bi-level decision-making techniques. Section 5 discusses the principal applications of fuzzy bi-level decision-making techniques. Our comprehensive analysis and main findings are given in Section 6.

\section{Basic Fuzzy Bi-level Decision-Making}

This section reviews the development of techniques for solving a category of fuzzy bi-level decision-making 
problems in which only one decision entity is involved at each decision level that attempts to optimize a unique objective; this category of problems can be termed basic fuzzy bi-level decision problems.

A general fuzzy linear bi-level decision problem (in the linear form) can be described as Definition 1.

Definition 1. [13, 14] For $x \in X \subset R^{p}, y \in Y \subset R^{q}$, and $F, f: R^{p} \times R^{q} \rightarrow F(R)$, a general fuzzy linear bilevel decision problem is defined as follows:

$$
\begin{aligned}
& \min _{x \in X} F(x, y)=\widetilde{c}_{1} x+\widetilde{d}_{1} y \quad \text { (1st level, leader) } \\
& \text { s.t. } \widetilde{A}_{1} x+\widetilde{B}_{1} y \leq \widetilde{b}_{1},
\end{aligned}
$$

where, for each $x$ given by the leader, $y$ solves (1c-1d)

$$
\begin{aligned}
& \min _{y \in Y} f(x, y)=\widetilde{c}_{2} x+\widetilde{d}_{2} y \quad \text { (2nd level, follower) } \\
& \text { s.t. } \widetilde{A}_{2} x+\widetilde{B}_{2} y \leq \widetilde{b}_{2}
\end{aligned}
$$

where $\widetilde{c}_{1}, \widetilde{c}_{2} \in F^{p}(R), \quad \widetilde{d}_{1}, \widetilde{d}_{2} \in F^{q}(R), \quad \widetilde{b}_{1} \in F^{m}(R)$, $\widetilde{b}_{2} \in F^{n}(R) \quad, \quad \widetilde{A}_{1} \in F^{m \times p}(R) \quad, \quad \widetilde{B}_{1} \in F^{m \times q}(R)$, $\widetilde{A}_{2} \in F^{n \times p}(R), \quad \widetilde{B}_{2} \in F^{n \times q}(R), F(R)$ is the set of all finite fuzzy numbers. The sets $X$ and $Y$ place additional restrictions on the decision variables, such as upper and lower bounds or integrality requirements [15].

The original appearance of fuzzy bi-level decisionmaking was proposed by Sakawa et al. [16] on a linear bi-level decision model with fuzzy parameters. The authors developed an interactive fuzzy programming approach for solving the problem. They then extended the interactive fuzzy programming approach to solve fuzzy linear bi-level fractional decision problems [17]. Sakawa, et al. [18, 19] also used the interactive fuzzy programming approach and genetic algorithms to solve fuzzy bi-level 0-1 decision problems and fuzzy bi-level nonconvex decision problems. In recent years, Pramanik [20] proposed a fuzzy goal programming approach to solve fuzzy linear bi-level decision problems. Biswas and Bose [21] developed a fuzzy goal programming approach for solving quadratic bi-level decision problems with fuzzy parameters that are only involved in constraint conditions. In general, these fuzzy approaches can be used to find solutions to a range of fuzzy bi-level decision problems. However, these methods are limited to solving fuzzy bi-level decision problems in special situations where decision entities from different levels share the same constraint conditions and prefer to cooperate with one another.
Many researchers extended traditional bi-level programming methods to solve fuzzy bi-level decision problems. Zhang and $\mathrm{Lu}$ [13] developed an approximation Kuhn-Tucker approach to solve fuzzy linear bi-level decision problems. They also presented an approximation Kth-Best algorithm and an approximation branch and bound algorithm to solve the fuzzy linear bi-level decision problem [22, 23]. Gao et al. [24] proposed a programmable $\lambda$-cut approximation algorithm to solve a $\lambda$-cut set based fuzzy goal bi-level decision problem. Budnitzki [25] used the selection function approach and a modified version of the $K$ thBest algorithm to solve a fuzzy linear bi-level decision problem. Katagiri et al. [26] defined Stackelberg solutions for fuzzy linear bi-level decision problems and then transformed fuzzy problems into deterministic linear or nonlinear bi-level decision problems that can be exactly solved by conventional bi-level solution methods. Furthermore, Zhang et al. [27] presented a fuzzy linear tri-level decision problem, and extended the linear tri-level $K$ th-Best algorithm proposed by Zhang et al. [28] to solve the problem based on $\lambda$-cut of a fuzzy set. Clearly, these solution methods are mainly for solving linear form of fuzzy bi-level and tri-level decision-making.

\section{Fuzzy Bi-level Decision-Making with Multiple Optima}

In a fuzzy bi-level decision problem, multiple optima may exist at each decision level:

(1) The leader or follower may have multiple and conflicting objectives, and this is a fuzzy bi-level multi-objective (FBLMO) decision problem;

(2) Multiple decision entities may exist at the lower (follower) level, and this is known as a fuzzy bilevel multi-follower (FBLMF) decision problem.

(3) Multiple decision entities may exist at the upper (leader) level, this is a fuzzy bi-level multi-leader (FBLML) decision problem.

Fuzzy bi-level decision-making with multiple optima has attracted numerous studies on decision models and solution methods.

In terms of research on FBLMO decision-making, a general linear FBLMO decision problem can be described as Definition 2. 
Definition 2. [29] For $x \in X \subset R^{p}, y \in Y \subset R^{q}$, and $F: R^{p} \times R^{q} \rightarrow F^{M}(R) \quad, \quad f: R^{p} \times R^{q} \rightarrow F^{N}(R) \quad, \quad a$ general linear FBLMO decision problem is formulated as follows:

$\min _{x \in X} F(x, y)=\left(\widetilde{c}_{11} x+\widetilde{d}_{11} y, \widetilde{c}_{12} x+\widetilde{d}_{12} y, \ldots, \widetilde{c}_{1 M} x+\widetilde{d}_{1 M} y\right)$

(1st level, leader)

s.t. $\widetilde{A}_{1} x+\widetilde{B}_{1} y \leq \widetilde{b}_{1}$, 2d)

where, for each $x$ given by the leader, $y$ solves (2c-

$$
\begin{aligned}
& \min _{y \in Y} f(x, y)= \\
& \left(\widetilde{c}_{21} x+\widetilde{d}_{21} y, \widetilde{c}_{22} x+\widetilde{d}_{22} y, \ldots, \widetilde{c}_{2 N} x+\tilde{d}_{2 N} y\right) \\
& \text { s.t. } \tilde{A}_{2} x+\widetilde{B}_{2} y \leq \widetilde{b}_{2}, \quad \text { (2nd level, follower) }
\end{aligned}
$$

where $\widetilde{c}_{1 i}, \widetilde{c}_{2 j} \in F^{p}(R), \widetilde{d}_{1 i}, \widetilde{d}_{2 j} \in F^{q}(R), i=1,2, \ldots, M$, $j=1,2, \ldots, N \quad, \quad \widetilde{b}_{1} \in F^{m}(R) \quad, \quad \widetilde{b}_{2} \in F^{n}(R)$, $\widetilde{A}_{1} \in F^{m \times p}(R) \quad, \quad \widetilde{B}_{1} \in F^{m \times q}(R) \quad, \quad \widetilde{A}_{2} \in F^{n \times p}(R)$, $\widetilde{B}_{2} \in F^{n \times q}(R), F(R)$ is the set of all finite fuzzy numbers. The sets $X$ and $Y$ place additional restrictions on the decision variables, such as upper and lower bounds or integrality requirements [15].

Zhang et al. [29] presented the linear FBLMO decision problem as shown in Definition 2 and developed an approximation Branch-and-Bound (BB) algorithm to solve it. Gao et al. [30] proposed a $\lambda$-cut and goalprogramming-based algorithm to solve the linear FBLMO decision problems as well. Pramanik and Dey [31] addressed a fuzzy goal programming approach for solving a linear FBLMO decision problem in which decision entities from different decision levels share the same constraints and prefer to cooperate with one another. Since the fuzzy goal programming approach does not follow the classical solution concepts of bilevel decision-making and neglects the upper-level decisions, Baky et al. [32] designed an extended fuzzy goal programming algorithm for solving the FBLMO problem.

For FBLMF decision-making, a general linear FBLMF decision problem can be described as Definition 3 based on related research in $[33,34]$.

Definition 3. [33, 34] For $x \in X \subset R^{p}, y_{i} \in Y_{i} \subset R^{q_{i}}$, and $F, f_{i}: R^{p} \times R^{q_{1}} \times \ldots \times R^{q_{k}} \rightarrow F(R), i=1,2, \ldots, k, a$ general linear FBLMF decision problem in which one leader and $k$ followers are involved can be written as:

$$
\begin{aligned}
& \min _{x \in X} F\left(x, y_{1}, \ldots, y_{k}\right)=\widetilde{c} x+\sum_{i=1}^{k} \widetilde{d}_{i} y_{i} \text { (1st level, leader) } \\
& \text { s.t. } \widetilde{A} x+\sum_{i=1}^{k} \widetilde{B}_{i} y \leq \widetilde{b}
\end{aligned}
$$

where, for each $x$ given, $y_{i}$ solves the ith follower's problem (3c-3d)

$$
\begin{aligned}
& \min _{y_{i} \in Y_{i}} f_{i}\left(x, y_{1}, \ldots, y_{k}\right)=\widetilde{c}_{i} x+\sum_{s=1}^{k} \widetilde{d}_{i s} y_{s} \\
& \text { (2nd level, the ith follower) (3c) } \\
& \begin{array}{ll}
\text { s.t. } \widetilde{A}_{i} x+\sum_{s=1}^{k} \widetilde{B}_{i s} y_{s} \leq \widetilde{b}_{i},
\end{array}
\end{aligned}
$$

where $\tilde{c}, \widetilde{c}_{i} \in F^{p}(R), \quad \tilde{d}_{i} \in F^{q_{i}}(R), \quad \tilde{d}_{i s} \in F^{q_{s}}(R)$, $\widetilde{b} \in F^{m}(R) \quad, \quad \widetilde{b}_{i} \in F^{n_{i}}(R) \quad, \quad \tilde{A} \in F^{m \times p}(R) \quad$, $\widetilde{B}_{i} \in F^{m \times q_{i}}(R), \quad \widetilde{A}_{i} \in F^{n_{i} \times p}(R), \quad \widetilde{B}_{i s} \in F^{n_{i} \times q_{s}}(R)$, $i, s=1,2, \ldots, k, F(R)$ is the set of all finite fuzzy numbers. The sets $X$ and $Y$ place additional restrictions on the decision variables, such as upper and lower bounds or integrality requirements [15]. It can be seen in Definition 3 that followers need to consider the decision results of their counterparts as references when making their individual decisions, in view of the decision given by the leader.

Gao et al. [33] focused on problems of fuzzy linear bilevel decision-making with multiple followers who share a common objective but have different constraints. The authors developed a partial swarm optimization (PSO) algorithm for solving these problem based on the ranking relationship among fuzzy sets defined by cut-set and satisfactory degree. Gao et al. [34] also studied a linear FBLMF decision problem in which multiple followers share the common constraints and developed a PSO algorithm to solve the problem. Gao and Liu [35] integrated fuzzy simulation, neural network and genetic algorithm to produce a hybrid intelligent algorithm for solving a nonlinear FBLMF problem.

In order to solve fuzzy bi-level multi-objective multi-follower (FBLMOMF) decision problems, Zhang et al. [36] proposed a set of linear models and developed an extended BB algorithm. Based on it, Zhang et al. [37] developed an approximation BB algorithm to solve a linear FBLMOMF decision problem with a partial cooperative relationship between multiple followers. Zhang et al. [38] also proposed a set of decision models to describe complex FBLMOMF decision issues and presented an approximation $\mathrm{BB}$ algorithm to solve a 
linear FBLMOMF decision problem in which focusing followers share the same decision variables. Zhang and $\mathrm{Lu}$ [39] presented an approximation Kth-Best algorithm to solve a linear FBLMOMF decision problem with a cooperative relationship between multiple followers. These developments provide a strong foundation for fuzzy bi-level decision-making techniques in various situations.

\section{Fuzzy Random Bi-level Decision-Making}

Fuzziness and randomness are two main sources of uncertainty [40]. Bi-level decision-making may appear in not only a fuzzy situation but also a random environment, and this is known as fuzzy random bilevel decision-making. Related parameters in relation to fuzzy random bi-level decision-making are called fuzzy random variables, which are introduced as random variables whose values are not real, but fuzzy numbers, and subsequently redefined as a particular kind of fuzzy sets [41]. With respect to Definition 1, if the coefficients of decision variables are characterized by fuzzy random variables, the fuzzy bi-level program (1) is known as a fuzzy random bi-level decision problem.

In recent years, attention has been increasingly paid to fuzzy random bi-level decision-making. Sakawa et al. [42] considered a linear bi-level decision problem under a fuzzy random environment and transformed the problem into a deterministic bi-level decision problem through level sets and probability maximization for finding a Stackelberg solution. Sakawa et al. [43] also used another different method based on expectation optimization using possibility and necessity to reduce a fuzzy random linear bi-level decision problem to a deterministic bi-level decision problem for solving. Ren and Wang [44] transformed a fuzzy random linear bilevel decision problem into a deterministic bi-level multi-objective decision problem using level sets and expectation optimization, and found a optimistic Stackelberg solution to the considered problem. Ren et al. [45] and Ren and Wang [46] developed interval programming approaches for solving fuzzy random linear bi-level decision problems. Katagiri et al. [47] introduced the concept of value at risk and possibility theory, and transformed a fuzzy random bi-level decision problem into a set of deterministic problems that can be solved by nonlinear bi-level decisionmaking techniques.

\section{Applications of Fuzzy Bi-level Decision- Making Techniques}

Fuzzy bi-level decision-making techniques have been widely applied to handle decentralized management problems in the real world that often appear in imprecise and ambiguous environments. These applications largely fall into the following four domains: (1) supply chain and logistics management; (2) planning and scheduling operations; (3) resource allocation in hierarchy; and (4) energy management and environment protection.

\subsection{Supply chain and logistics management}

In terms of applications in supply chain and logistics management, Gao et al. [48] established a fuzzy bi-level decision model for buyers and vendors in a pricing supply chain that some coefficients are generally obtained from experiments and cannot be defined as crisp numbers. A PSO algorithm was developed to solve the resulting model. Sun and Gao [49] proposed a bilevel decision model to handle pricing and production decisions with fuzzy demand and fuzzy cost parameters. The upper level (leader) aims to determine the optimal price and production quantity with capacity constraints, whereas the lower level (follower) problem tries to structure the distribution pattern of customers with the aim of satisfying the demand at minimum cost. Ji and Shao [50] addressed a bi-level decision model for a newsboy problem with fuzzy demands and quantity discounts in a hierarchical decision system, in which manufacturer at the upper level decides the wholesale prices of newspaper in order to maximize its profit, while retailers at the lower level decide ordering quantities of newspaper in order to maximize their own expected profits. Zhang et al. [51] applied fuzzy bi-level optimization techniques to model and support bidding decision-making between the generating company and the market operator in electricity markets. Lan et al. [52] studied an optimal taxation problem with asymmetric information in which the government and the monopolist are principals while the consumer is their agent. The authors formulated this problem as a fuzzy nonlinear bi-level decision model with the purpose of maximizing the expected social welfare and the monopolist's expected welfare under an incentive feasible mechanism. 


\subsection{Planning and scheduling operations}

For planning and scheduling operations in a decentralized manner, Abass [53] presented a fuzzy bilevel programming approach to solve a flow shop scheduling problem with fuzzy processing times in a hierarchy of two decision entities, in which the shop owner at the higher level assigns jobs to machines in order to minimize the flow time, while the customer at the lower level decides on a job schedule in order to minimize the makespan. Konur and Golias [54] studied scheduling of inbound trucks at inbound doors of a cross-dock facility under truck arrival time uncertainty. The authors formulated this problem as pessimistic and optimistic bi-level decision problems respectively in which the uncertain truck arrival time is handled by fuzzy number with a triangular distribution. Also, the authors developed a genetic algorithm to solve the bilevel pessimistic and optimistic formulations.

\subsection{Resource allocation in hierarchy}

In regard to handling resource allocation in hierarchy, Gang et al. [55] focused on a multi-project resource allocation problem using bi-level optimization. In this problem, the company manager at the upper level aims to allocate the company's resources to multiple projects to achieve the lowest cost that include resource costs and a tardiness penalty, while each project manager at the lower level attempts to schedule their resourceconstrained project with the minimization of project duration. The authors applied a fuzzy random bi-level decision model to describe this problem in which fuzzy random variables are used to model uncertain activity durations and resource costs. Xu and Gang [56] studied a material flow traffic assignment problem with fuzzy random variables in a large-scale construction project. A fuzzy random bi-level multi-objective decision model was established in which the transportation time and cost in each arc are considered as fuzzy random variables, and the authors developed a PSO algorithm to solve the resulting model.

\subsection{Energy management and environment protection}

Fuzzy bi-level decision-making techniques have been also applied to handle energy management problems in relation to the consumption of resources and the generation of waste. Aviso et al. [57] developed a fuzzy bi-level optimization model to explore the effect of charging fees for the purchase of freshwater and the treatment of wastewater in optimizing the water exchange network of plants in an eco-industrial park (EIP). Tan et al. [58] extended the optimization model developed by Aviso et al. [57] to a new fuzzy bi-level decision model by modifying the role of the EIP authority to include water regeneration and redistribution via a centralized hub, and found a reasonable compromise between the EIP authority's desire to minimize fresh water usage and the participating companies' desire to minimize costs. Xu et al. [59] developed a fuzzy random bi-level decision model for tackling a regional water resources allocation problem on the basis of water rights distribution in a river basin. The bi-level decision model targets to optimize the total benefit of the society and the economic benefit of each subarea respectively for the upper and lower levels.

From the aspect of environment protection and risk analysis, Gan and $\mathrm{Xu}$ [60] considered a problem of hedging against seismic risk through the retrofit of transportation systems in large-scale construction projects (LSCP). They formulated this problem as a fuzzy random multi-objective bi-level decision model with the objectives of the retrofit costs and the benefits on two separate levels. Xu et al. [61] focused on a bilevel decision problem of hazmat transportation network design, which considers an location problem of emergency response teams. The authority at the upper level designs the transportation network to minimize the total transportation risk, while the carriers at the lower level choose their routes with the aim of minimizing the total transportation cost. The problem was formulated as fuzzy bi-level optimization model in which a complex fuzzy variable was used to model transportation risk, and then an improved artificial bee colony algorithm with priority-based encoding was applied to find an optimal solution to the resulting model. For the sake of optimizing regional economies, social employment, and water quality protection, $\mathrm{Xu}$ et al. [62] proposed a fuzzy random multi-objective bi-level optimization model for constructed wetland planning in which fuzzy random variables are used to describe uncertainties in the system. Yao et al. [63] gave a fuzzy bi-level multiobjective optimization model to handle a stone resource assignment problem with the aim of decreasing dust and waste water emissions. A fuzzy simulation-based 
improved simulated annealing (FS-ISA) algorithm was developed to find the Pareto optimal solutions.

In addition to these fuzzy bi-level decision-making applications in a range of domains, related decision support systems (software) have been developed to assist decision markers in solving fuzzy bi-level decision problems. Gao et al. [24] developed a fuzzy bilevel decision support system software based on fuzzy goal bi-level decision-making techniques, as shown in Fig. 1, and applied the software to handle a problem of traffic management and risk analysis in a disaster area. The authors also applied the fuzzy bi-level decision support system to deal with a manufacturer-retailer decision problem in an uncertain marketing situation [64].

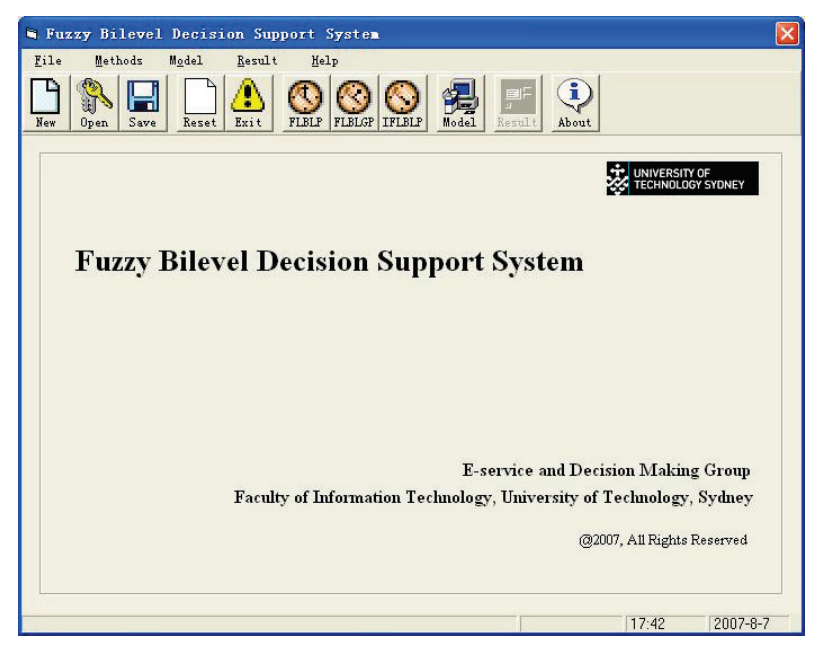

Fig. 1. The main interface of the fuzzy bi-level decision support system developed by Gao et al. [24, 64]

\section{Comprehensive Analysis and Prospective Research Topics}

In this paper, we have reported the new developments in fuzzy bi-level decision-making techniques including basic fuzzy bi-level decision-making, fuzzy bi-level decision-making with multiple optima, fuzzy random bi-level decision-making, and their applications in handling real-world cases through a comprehensive review. This review demonstrates the following features of fuzzy bi-level decision-making study:

(1) A number of solution approaches/algorithms have been developed to solve a range of fuzzy bi-level decision problems. The majority of the solution approaches are based on the idea that transforms a fuzzy bi-level decision problem into a deterministic decision problem for solving in the first place.

(2) Motivated by the constant complexity of uncertain situations, an increasing number of papers are devoted to fuzzy bi-level decision-making with multiple optima and fuzzy random bi-level decision-making. A range of advanced fuzzy bilevel decision models and effective solution algorithms have been developed to handle these complex situations.

(3) Fuzzy bi-level decision-making techniques have been widely applied to handle decentralized management and optimization problems under uncertainty in various domains. The application domains and fuzzy bi-level decision-making techniques used in applications are summarized in Table 1, which shows the number of each category of fuzzy bi-level decision-making techniques applied in each domain.

Table 1. Summary of fuzzy bi-level decision-making techniques in each application domain

\begin{tabular}{|c|c|c|c|c|}
\hline \multirow[b]{2}{*}{ Application domains } & \multicolumn{4}{|c|}{ Decision-making techniques } \\
\hline & $\begin{array}{c}\text { Basic fuzzy bi-level } \\
\text { decision-making }\end{array}$ & $\begin{array}{c}\text { Fuzzy bi-level decision-making } \\
\text { with multiple optima }\end{array}$ & $\begin{array}{c}\text { Fuzzy random bi-level } \\
\text { decision-making }\end{array}$ & $\begin{array}{l}\text { No. of listed } \\
\text { references }\end{array}$ \\
\hline Supply chain and logistics management & 4 & & & 4 \\
\hline Planning and scheduling operations & 2 & & & 2 \\
\hline Resource allocation in hierarchy & & & 2 & 2 \\
\hline $\begin{array}{l}\text { Energy management and environment } \\
\text { protection }\end{array}$ & 4 & 2 & 3 & 9 \\
\hline Total & 10 & 2 & 5 & 17 \\
\hline
\end{tabular}


Although fuzzy bi-level decision-making techniques have attracted much attention, several evident challenges still require further research:

(1) Computational complexity is a crucial issue in solving fuzzy bi-level decision problems. Although many solution algorithms have been developed, it is necessary and urgent to develop much more efficient algorithms, e.g. heuristic algorithms, for solving much more general formulations, e.g. nonlinear problems with complex membership functions, and solving large-scale versions in practically reasonable computational time. Moreover, fully fuzzy bi-level decision problems, in which both coefficients and variables are characterized by fuzzy numbers, are also an emerging research topic with respect to computational complexity and need to be explored in depth.

(2) Bi-level decision problems nowadays often appear in a highly uncertain decision environment, thus, this requires further research on how to wisely model such uncertain problems by means of a fuzzy bi-level decision support system. Many bi-level decision problems under fuzziness in the real world cannot be modeled as mathematical programming formulations. Consequently, it is a challenge to break through mathematical programs and integrate the principle of fuzzy bi-level decision-making with other decision-making techniques to handle a much wider range of decision problems under uncertainty.

(3) A new direction in handling bi-level decisionmaking is data-driven modeling in the current age of big data. How to implement data-driven bi-level decision-making under uncertainty using data analytical techniques and cognitive decisionmaking methods [65] can be considered as a future research issue.

Two important features of the paper clearly distinguish it from previous survey papers in the bi-level/multilevel decision-making area. On the one hand, in contrast to previous survey papers that only review deterministic bi-level decision-making techniques, this survey targets the latest bi-level decision-making using fuzzy techniques and their comprehensive development. On the other hand, apart from theoretical research developments, this survey systematically examines the real-world application developments of fuzzy bi-level decision-making techniques in different domains. We believe that this paper provides researchers and practitioners with the state-of-the-art knowledge on the development of fuzzy bi-level decision-making approaches and gives guidelines about how to apply fuzzy bi-level decision-making techniques to deal with various decision activities under uncertainty in realworld applications.

\section{Acknowledgements}

This work is supported by the Australian Research Council (ARC) under discovery grant DP140101366.

\section{References}

1. H.V. Stackelberg, The Theory of Market Economy, Oxford University Press, Oxford, 1952.

2. J. Bracken, J.T. McGill, Mathematical programs with optimization problems in the constraints, Operations Research, 21 (1973) 37-44.

3. J. Lu, G. Zhang, J. Montero, L. Garmendia, Multifollower trilevel decision making models and system, IEEE Transactions on Industrial Informatics, 8 (2012) 974-985

4. J. Lu, C. Shi, G. Zhang, On bilevel multi-follower decision making: General framework and solutions, Information Sciences, 176 (2006) 1607-1627.

5. M. Sakawa, I. Nishizaki, Interactive fuzzy programming for multi-level programming problems: a review, International Journal of Multicriteria Decision Making, 2 (2012) 241-266.

6. J. Han, J. Lu, Y. Hu, G. Zhang, Tri-level decision-making with multiple followers: Model, algorithm and case study, Information Sciences, 311 (2015) 182-204.

7. V.V. Kalashnikov, S. Dempe, G.A. Pérez-Valdés, N.I. Kalashnykova, J.-F. Camacho-Vallejo, Bilevel programming and applications, Mathematical Problems in Engineering, 2015 (2015) 1-16.

8. J. Lu, J. Han, Y. Hu, G. Zhang, Multilevel decisionmaking: A survey, Information Sciences, 346 (2016) 463 487.

9. O. Ben-Ayed, Bilevel linear programming, Computers \& Operations Research, 20 (1993) 485-501.

10. L. Vicente, P. Calamai, Bilevel and multilevel programming: A bibliography review, Journal of Global Optimization, 5 (1994) 291-306.

11. B. Colson, P. Marcotte, G. Savard, Bilevel programming: A survey, 4OR, 3 (2005) 87-107.

12. B. Colson, P. Marcotte, G. Savard, An overview of bilevel optimization, Annals of Operations Research, 153 (2007) 235-256. 
13. G. Zhang, J. Lu, The definition of optimal solution and an extended Kuhn-Tucker approach for fuzzy linear bilevel programming, IEEE Computational Intelligence Bulletin, 2 (2005) 1-7.

14. G. Zhang, J. Lu, Y. Gao, Multi-Level Decision Making: Models, Methods and Applications, Springer, Berlin, 2015.

15. J.F. Bard, Practical Bilevel Optimization: Algorithms and Applications, Kluwer Academic Publishers, Dordrecht, The Netherlands, 1998.

16. M. Sakawa, I. Nishizaki, Y. Uemura, Interactive fuzzy programming for multi-level linear programming problems with fuzzy parameters, Fuzzy Sets and Systems, 109 (2000) 3-19.

17. M. Sakawa, I. Nishizaki, Y. Uemura, Interactive fuzzy programming for two-level linear fractional programming problems with fuzzy parameters, Fuzzy Sets and Systems, 115 (2000) 93-103.

18. M. Sakawa, I. Nishizaki, M. Hitaka, Interactive fuzzy programming for multi-level 0-1 programming problems with fuzzy parameters through genetic algorithms, Fuzzy Sets and Systems, 117 (2001) 95-111.

19. M. Sakawa, I. Nishizaki, Interactive fuzzy programming for two-level nonconvex programming problems with fuzzy parameters through genetic algorithms, Fuzzy Sets and Systems, 127 (2002) 185-197.

20. S. Pramanik, Bilevel programming problem with fuzzy parameters: A fuzzy goal programing approach, Journal of Applied Quantitative Methods, 7 (2012) 9-24.

21. A. Biswas, K. Bose, A fuzzy programming approach for solving quadratic bilevel programming problems with fuzzy resource constraints, International Journal of Operational Research, 12 (2011) 142-156.

22. G. Zhang, J. Lu, Model and approach of fuzzy bi-level decision making for logistics planning problem, Journal of Enterprise Information Management, 20 (2007) 178197.

23. G. Zhang, J. Lu, T. Dillon, Fuzzy linear bilevel optimization: Solution concepts, approaches and applications, in Fuzzy Logic, Springer Berlin Heidelberg, 2007, pp. 351-379.

24. Y. Gao, G. Zhang, J. Lu, T. Dillon, X. Zeng, A $\lambda$-cutapproximate algorithm for goal-based bilevel risk management systems, International Journal of Information Technology and Decision Making, 7 (2008) 589-610.

25. A. Budnitzki, The solution approach to linear fuzzy bilevel optimization problems, Optimization, (2013) 115.

26. H. Katagiri, K. Kato, T. Uno, Possibilistic Stackelberg solutions to bilevel linear programming problems with fuzzy parameters, in Joint IFSA World Congress and NAFIPS Annual Meeting, 2013, pp. 134-139.

27. Z. Zhang, G. Zhang, J. Lu, C. Guo, A fuzzy tri-level decision making algorithm and its application in supply chain, in The 8th conference of the European Society for
Fuzzy Logic and Technology, Atlantis Press, 2013, pp. 154-160

28. G. Zhang, J. Lu, J. Montero, Y. Zeng, Model, Solution concept and the Kth-best algorithm for linear tri-level programming, Information Sciences, 180 (2010) 481-492.

29. G. Zhang, J. Lu, T. Dillon, Decentralized multi-objective bilevel decision making with fuzzy demands, KnowledgeBased Systems, 20 (2007) 495-507.

30. Y. Gao, G. Zhang, J. Ma, J. Lu, A $\lambda$-cut and goal programming based algorithm for fuzzy linear multiple objective bi-level optimization, IEEE Transactions on Fuzzy Systems, 18 (2010) 1-13.

31. S. Pramanik, P.P. Dey, Bi-level multi-objective programming problem with fuzzy parameters, International Journal of Computer Applications, 30 (2011) 13-20.

32. I. Baky, M. Eid, M. El Sayed, Bi-level multi-objective programming problem with fuzzy demands: a fuzzy goal programming algorithm, OPSEARCH, 51 (2014) 280296.

33. Y. Gao, G. Zhang, J. Lu, A particle swarm optimization based algorithm for fuzzy bilevel decision making with objective-shared followers, in Simulated Evolution and Learning, Springer Berlin Heidelberg, 2008, pp. 190-199.

34. Y. Gao, G. Zhang, J. Lu, A particle swarm optimization based algorithm for fuzzy bilevel decision making with constraints-shared followers, in The ACM symposium on Applied Computing, ACM, Honolulu, Hawaii, 2009, pp. 1075-1079.

35. J. Gao, B. Liu, Fuzzy multilevel programming with a hybrid intelligent algorithm, Computers \& Mathematics with Applications, 49 (2005) 1539-1548.

36. G. Zhang, J. Lu, T. Dillon, Models and algorithm for fuzzy multi-objective multi-follower linear bilevel programming, in IEEE International Fuzzy Systems Conference, 2007, pp. 1-6.

37. G. Zhang, J. Lu, Y. Gao, An algorithm for fuzzy multiobjective multi-follower partial cooperative bilevel programming, Journal of Intelligent \& Fuzzy Systems, 19 (2008) 303-319.

38. G. Zhang, J. Lu, Y. Gao, Fuzzy bilevel programming: Multi-objective and multi-follower with shared variables, International Journal of Uncertainty, Fuzziness and Knowledge-Based Systems, 16 (2008) 105-133.

39. G. Zhang, J. Lu, Fuzzy bilevel programming with multiple objectives and cooperative multiple followers, Journal of Global Optimization, 47 (2010) 403-419.

40. A.F. Shapiro, Fuzzy random variables, Insurance: Mathematics and Economics, 44 (2009) 307-314.

41. H. Kwakernaak, Fuzzy random variables-I. definitions and theorems, Information Sciences, 15 (1978) 1-29.

42. M. Sakawa, H. Katagiri, T. Matsui, Stackelberg solutions for fuzzy random bilevel linear programming through level sets and probability maximization, Operational Research, 12 (2012) 271-286.

43. M. Sakawa, H. Katagiri, T. Matsui, Fuzzy random bilevel linear programming through expectation optimization 
using possibility and necessity, International Journal Machine Learning and Cybernetics, 3 (2012) 183-192.

44. A. Ren, Y. Wang, Optimistic Stackelberg solutions to bilevel linear programming with fuzzy random variable coefficients, Knowledge-Based Systems, 67 (2014) 206217.

45. A. Ren, Y. Wang, X. Xue, An interval programming approach for the bilevel linear programming problem under fuzzy random environments, Soft Computing, 18 (2014) 995-1009.

46. A. Ren, Y. Wang, An interval approach based on expectation optimization for fuzzy random bilevel linear programming problems, Journal of the Operational Research Society, (2015) 1-11.

47. H. Katagiri, T. Uno, K. Kato, H. Tsuda, H. Tsubaki, Random fuzzy bilevel linear programming through possibility-based value at risk model, International Journal Machine Learning and Cybernetics, 5 (2014) 211-224.

48. Y. Gao, G. Zhang, J. Lu, H.-M. Wee, A fuzzy bi-level pricing model and a PSO based algorithm in supply chains, in Neural Information Processing, Springer Berlin Heidelberg, 2009, pp. 226-233.

49. H. Sun, Z. Gao, Bilevel model for pricing and production planning decision with fuzzy parameters, Journal of Systems Science \& Information, 2 (2004) 335-344.

50. X. Ji, Z. Shao, Model and algorithm for bilevel newsboy problem with fuzzy demands and discounts, Applied Mathematics and Computation, 172 (2006) 163-174.

51. G. Zhang, G. Zhang, Y. Gao, J. Lu, A fuzzy bilevel model and a PSO-based algorithm for day-ahead electricity market strategy making, in Knowledge-Based and Intelligent Information and Engineering Systems, Springer Berlin Heidelberg, 2009, pp. 736-744.

52. Y. Lan, R. Zhao, W. Tang, A bilevel fuzzy principalagent model for optimal nonlinear taxation problems, Fuzzy Optimization and Decision Making, 10 (2011) 211232.

53. S. Abass, Bilevel programming approach applied to the flow shop scheduling problem under fuzziness, Computational Management Science, 2 (2005) 279-293.

54. D. Konur, M.M. Golias, Analysis of different approaches to cross-dock truck scheduling with truck arrival time uncertainty, Computers \& Industrial Engineering, 65 (2013) 663-672.

55. J. Gang, J. Xu, Y. Xu, Multiproject resources allocation model under fuzzy random environment and its application to industrial equipment installation engineering, Journal of Applied Mathematics, (2013) 119.

56. J. Xu, J. Gang, Multi-objective bilevel construction material transportation scheduling in large-scale construction projects under a fuzzy random environment, Transportation Planning and Technology, 36 (2013) 352376.

57. K.B. Aviso, R.R. Tan, A.B. Culaba, J.B. Cruz Jr, Bi-level fuzzy optimization approach for water exchange in ecoindustrial parks, Process Safety \& Environmental Protection: Transactions of the Institution of Chemical Engineers Part B, 88 (2010) 31-40.

58. R.R. Tan, K.B. Aviso, J.B. Cruz Jr, A.B. Culaba, A note on an extended fuzzy bi-level optimization approach for water exchange in eco-industrial parks with hub topology, Process Safety \& Environmental Protection: Transactions of the Institution of Chemical Engineers Part B, 89 (2011) 106-111.

59. J. Xu, Y. Tu, Z. Zeng, Bilevel optimization of regional water resources allocation problem under fuzzy random environment, Journal of Water Resources Planning and Management, 139 (2012) 246-264.

60. L. Gan, J. Xu, Retrofitting transportation network using a fuzzy random multiobjective bilevel model to hedge against seismic risk, Abstract and Applied Analysis, 2014 (2014) 24.

61. J. Xu, J. Gang, X. Lei, Hazmats transportation network design model with emergency response under complex fuzzy environment, Mathematical Problems in Engineering, 2013 (2013) 1-22.

62. J. Xu, J. Ni, M. Zhang, Constructed wetland planningbased bilevel optimization model under fuzzy random environment: Case study of Chaohu Lake, Journal of Water Resources Planning and Management, 141 (2014) $1-18$.

63. L. Yao, J. Xu, F. Guo, A stone resource assignment model under the fuzzy environment, Mathematical Problems in Engineering, 2012 (2012) 1-26.

64. Y. Gao, G. Zhang, J. Lu, A fuzzy multi-objective bilevel decision support system, International Journal of Information Technology \& Decision Making, 8 (2009) 93-108.

65. L. Niu, J. Lu, G. Zhang, Cognition in Business Decision Support Systems, Springer, 2009. 